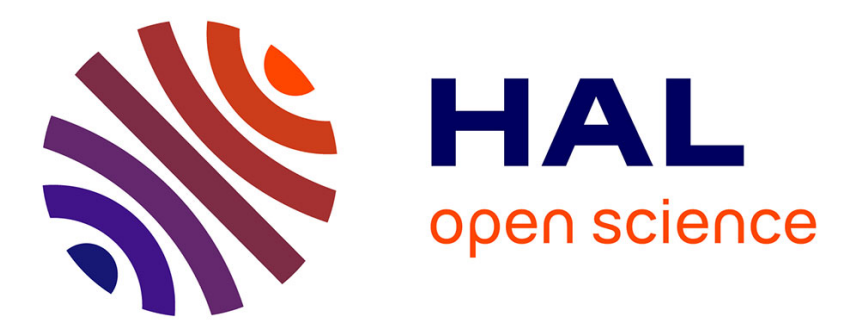

\title{
Search for the wobbling mode built on triaxial super deformation
}

S.W. Odegard, G.B. Hagemann, M. Bergstrom, B. Herskind, D.R. Jensen, G. Sletten, S. Tormanen, J. Wilson, P.O. Tjom, K. Spohr, et al.

\section{- To cite this version:}

S.W. Odegard, G.B. Hagemann, M. Bergstrom, B. Herskind, D.R. Jensen, et al.. Search for the wobbling mode built on triaxial super deformation. Nuclear Physics A, 2001, 682, pp.C427-C432. in2p3-00009885

\section{HAL Id: in2p3-00009885 \\ https://hal.in2p3.fr/in2p3-00009885}

Submitted on 9 Apr 2001

HAL is a multi-disciplinary open access archive for the deposit and dissemination of scientific research documents, whether they are published or not. The documents may come from teaching and research institutions in France or abroad, or from public or private research centers.
L'archive ouverte pluridisciplinaire HAL, est destinée au dépôt et à la diffusion de documents scientifiques de niveau recherche, publiés ou non, émanant des établissements d'enseignement et de recherche français ou étrangers, des laboratoires publics ou privés. 
Search for the wobbling mode built on triaxial super deformation*

S. W. Ødegârd ${ }^{\mathrm{a}, \mathrm{b}}$, G.B. Hagemann ${ }^{\mathrm{a}}$, M. Bergström ${ }^{a}$, B. Herskind ${ }^{\mathrm{a}}$, D. R. Jensen ${ }^{\mathrm{a}}$, G. Sletten ${ }^{a}$, S. Törmänen ${ }^{a}$, J. Wilson ${ }^{a}$, P.O. Tjøm ${ }^{b}$, K. Spohr ${ }^{c}$, H. Hübel ${ }^{d}$, A. Görgen ${ }^{d}$,

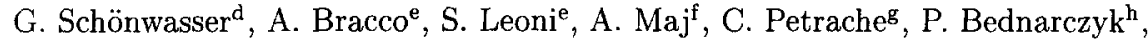
D. Curien ${ }^{\text {b }}$ and I. Hamamoto

${ }^{a}$ The Niels Bohr Institute, Blegdamsvej 17, DK-2100 Copenhagen $\varnothing$, Denmark

${ }^{b}$ Dept. of Physics, Univ. of Oslo, PB 1048 Blindern, N-0316 Oslo, Norway

${ }^{c}$ Department of Electronic Fngineering \& Physics, Univ. of Paisley, Scotland

${ }^{\mathrm{d} I n s t i t u t ~ f u ̈ r}$ Strahlen- und Kernphysik, University of Bonn, Germany

eDipartemento di Fisica and INFN, Sezione di Milano, Milano, Italy

${ }^{\mathrm{f}}$ Niewodniczanski Institute of Nuclear Physics, Krakow, Poland

gDipartemento di Fisica and INFN, Sezione di Padova, Padova, Italy

${ }^{\mathrm{h}}$ IReS, 23 rue du Loess, BP28 F-67037, Strasbourg, France

'Dept. of Mathematical Physics, LTH, University of Lund, Lund, Sweden

The nucleus ${ }^{163} \mathrm{Lu}$ is studied through the fusion evaporation reaction ${ }^{139} \mathrm{La}\left({ }^{29} \mathrm{Si}, 5 \mathrm{n}\right){ }^{163} \mathrm{Lu}$ with a beam energy of $152 \mathrm{MeV}$. The linking transitions between two presumably triaxial super deformed (TSD) bands have been studied. The possible assignment of the excited TSD band as a wobbling mode built on the yrast TSD band is discussed.

\section{INTRODUCTION}

Rotational bands based on the deformation driving $\pi i_{13 / 2}$ intruder configuration have heen ohserved in several even-N $T_{11}$ isotopes [1-3] and in odd-odd ${ }^{164} T_{11}$ where the $i_{13 / 2}$ proton is coupled to different neutron orbitals [4]. The quadrupole moment is only measured in ${ }^{163} \mathrm{Lu}\left(Q_{t} \approx 11 b\right)$ [1], however, the dynamical moments of inertia are very similar in all these bands, and larger than those of the bands built on the normal deformed (ND) structures.

For nuclei with $N \sim 94$ and $\mathrm{Z} \sim 71-72$, calculations predict stable triaxial shapes with large quadrupole deformation $\left(\varepsilon_{2} \sim 0.38\right.$ and $\left.\gamma \sim \pm 20^{\circ}\right)$ for all symmetry groups of parity and signature $[2,5]$. In Fig. 1 the potential energy surface of ${ }^{163} \mathrm{Lu}$ shows a rather deep

\footnotetext{
* This project has heen supported by the Danish Natural Science Foundation, the EU TMR project. nn ERBFMBICT980145, the Research Council of Norway, BMBF Germany and the Polish Committee for Scientific Research (KBN Grant No.2 P03B001 16)
} 
local minimum at $\epsilon, \gamma \approx 0.4,+20^{\circ}$, while the local minimum at $-20^{\circ}$ is poorly developed. At both local minima the $\pi i_{13 / 2}$ orbital is lowest in energy.

So far, no direct experimental evidence for the triaxiality of the observed TSD bands have been given, but one possible consequence of a rotating nucleus with a triaxial shape would be the existence of "wobbling bands" [6]. A favorable condition for the wobbling mode may be provided by nuclei in this region with triaxial SD bands (TSD) built on stably deformed triaxial minima, like the case shown in Fig. 1.

In an investigation of the isotopes ${ }^{163,164} \mathrm{Lu}$ with the Euroball array, a second band (TSD2) with similar properties as the previously known $i_{13 / 2}$ band (TSD1) has been observed in ${ }^{163} \mathrm{Lu}$ [7]. This second band was found to decay to TSD1, but no connections could be established. The new band is considered a candidate for a wobbling excitation built on TSD1.

\section{THE WOBBLING MODE}

The wobbling mode is a direct consequence of rotational motion of a triaxial body. In the high spin limit, with most of the spin aligned along one of the principal axes, the wobbling degree of freedom introduces sequences of bands with increasing number of wobbling quanta [6], and a characteristic decay pattern between the bands in competition with the in-band decay as shown in Fig. 2. In principle there will be a wobbling pattern built on each intrinsic excitation. An approximate expression for the excitation energies of the levels [6] is

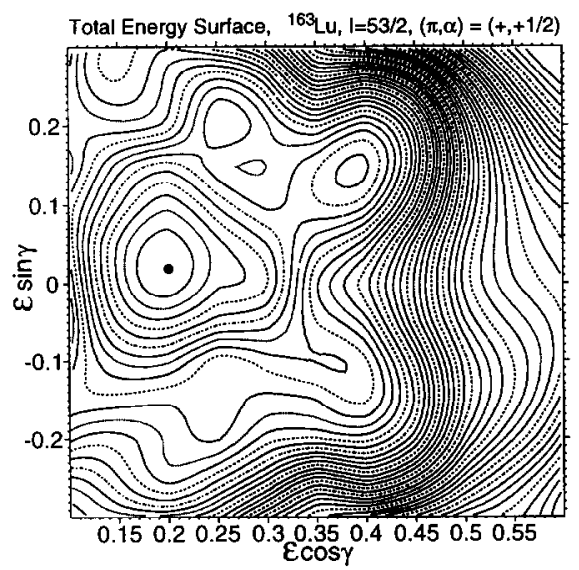

Figure 1. Total energy surface from Ultimate Cranker $[8,9]$ for spin $53 / 2$ in ${ }^{163} \mathrm{Lu}$ for $(\pi, \alpha)=(+,+1 / 2)$.

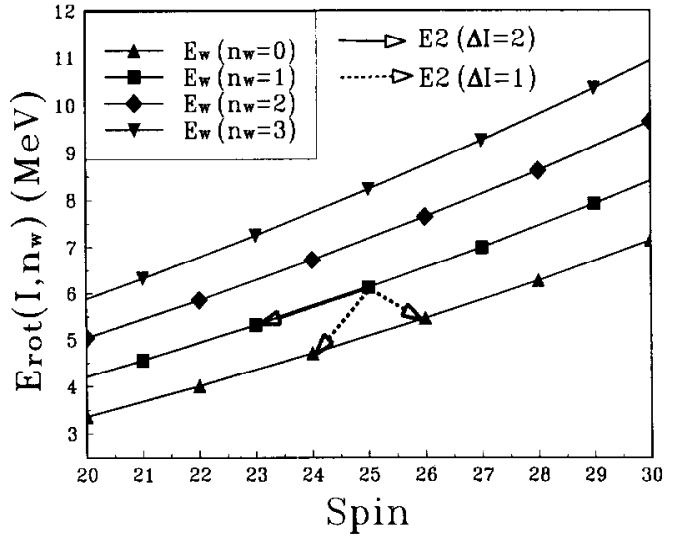

Figure 2. Wobbling pattern simulated with $\omega_{w o b} / \omega_{\text {rot }}=3$. 
$E_{R}\left(I, n_{w}\right)=\frac{I(I+1)}{2 J_{x}}+\hbar \omega_{w}\left(n_{w}+1 / 2\right)$

where the main rotation takes place around the axis with largest moment of inertia, $J_{x}$. The wobbling frequency which is determined by the different moments of inertia for the three principal axes is given [6] by $\hbar \omega_{w}=\hbar \omega_{\text {rot }} \sqrt{\left(J_{x}-J_{y}\right)\left(J_{x}-J_{z}\right) / J_{y} J_{z}}$ with $\hbar \omega_{\text {rot }}=I / J_{x}$. The integer $n_{w}=0,1,2, \ldots$ is the wobbling phonon number characterizing the wobbling excitations on the "yrast" $\left(n_{w}=0\right)$ states. The moments of inertia may be estimated within the hydro-dynamical model, which in the case of $\gamma \sim+20^{\circ}$ gives $\hbar \omega_{w} \approx 3 \hbar \omega_{\text {rot }}$, or with rigid moments of inertia, giving $\hbar \omega_{w}$ much closer to $\hbar \omega_{\text {rot }}$. The competition between the $\Delta I=1, \Delta n_{w}=1$ 'out of band' and $\Delta I=2, \Delta n_{w}=0$ 'in-band' $E 2$ transitions depends on the relevant transition energies and $B(E 2)$ values. The ratio of the average 'out-of-band' to the 'in-band' $B(E 2)$ values are estimated to be of the order of $1 / \mathrm{I}[6]$.

\section{EXPERIMENTAL RESULTS}

To find and investigate the nature of the linking transitions between TSD2 and TSD1, an experiment was done with Euroball IV in Strasbourg. At the time, 15 cluster detectors, 25 clover detectors, and 26 tapcred detectors werc used together with the BGO inner ball. With the ${ }^{139} \mathrm{La}\left({ }^{29} \mathrm{Si}, 5 \mathrm{n}\right){ }^{163} \mathrm{Lu}$ reaction and a beam energy of $152 \mathrm{MeV}$, approximately $2.4 \times 10^{9}$ events with 3 or more Compton suppressed $\gamma$-rays were collected and used in $3 \mathrm{D}$ and $4 \mathrm{D}$ coincidence analyses.

The preliminary analysis has extended TSD2 to both lower and higher spins, and revealed 7 connecting transitions to TSD1, see Fig. 3. A gated spectrum illustrating the linking transitions as well as the in-band transitions in TSD1 and TSD2 is shown in Fig. 4. The spectrum is a sum of double gates, with $\mathrm{x}$-gate in TSD1 $(315+386+450+515+639$ $\mathrm{keV})$ and $y$-gate is in TSD2 $(472+595+654+711+766+820+873+926 \mathrm{keV})$.

The relative population of TSD1 $(\sim 10 \%)$ and TSD2 $(\sim 2.5 \%)$ in ${ }^{163} \mathrm{Lu}$ indicates the spin assignment of TSD2, and implicitly $\Delta I=1$ for the connecting transitions. This may be seen from the level energies in Fig. 5 , in which the alternative solutions with $\Delta I=0$ and $\Delta I=2$ are marked with dashed symbols.

DCO ratios for three of the connecting transitions were obtained by sorting a matrix of energies detected in the two most forward rings of tapered and the most backward ring of the clusters along one axis and energies detected in the clovers along the other axis. In addition, two angular distribution matrices with energies detected in any angle along one axis and energies detected at forward or $90^{\circ}$ along the other axis, were sorted. The DCO ratios obtained, $\sim 0.4-0.5$, for the connecting transitions are compatible with the expected values for stretched dipole transitions or non-stretched $\Delta I=1$ quadrupole transitions. The very preliminary values of the ratio $W\left(90^{\circ}\right) / W\left(25^{\circ}\right)$ for three of the connecting transitions are not compatible with the expected ratio for any pure transitions of dipole or quadrupole type, thus hinting towards a mixing of E2/M1 multipolarities. In that case, the DCO values suggest two solutions, both with $\delta<0$, one with a $80 \%-100 \%$ E2 component and one with a $0 \%-20 \%$ E2 component. These solutions correspond to either $\mathrm{B}(\mathrm{E} 2)_{\text {out }} / \mathrm{B}(\mathrm{E} 2)_{\text {in }}$ and $\mathrm{B}(\mathrm{M} 1) / \mathrm{B}(\mathrm{E} 2)_{\text {in }}=\sim 0.23$ and $\sim 0.006 \mu_{N}^{2} / e^{2} b^{2}$, or $\mathrm{B}(\mathrm{E} 2)_{\text {out }} / \mathrm{B}(\mathrm{E} 2)_{\text {in }}$ and $\mathrm{B}(\mathrm{M} 1) / \mathrm{B}(\mathrm{E} 2)_{\text {in }}=\sim 0.025$ and $\sim 0.06 \mu_{N}^{2} / e^{2} b^{2}$, respectively. Pos- 


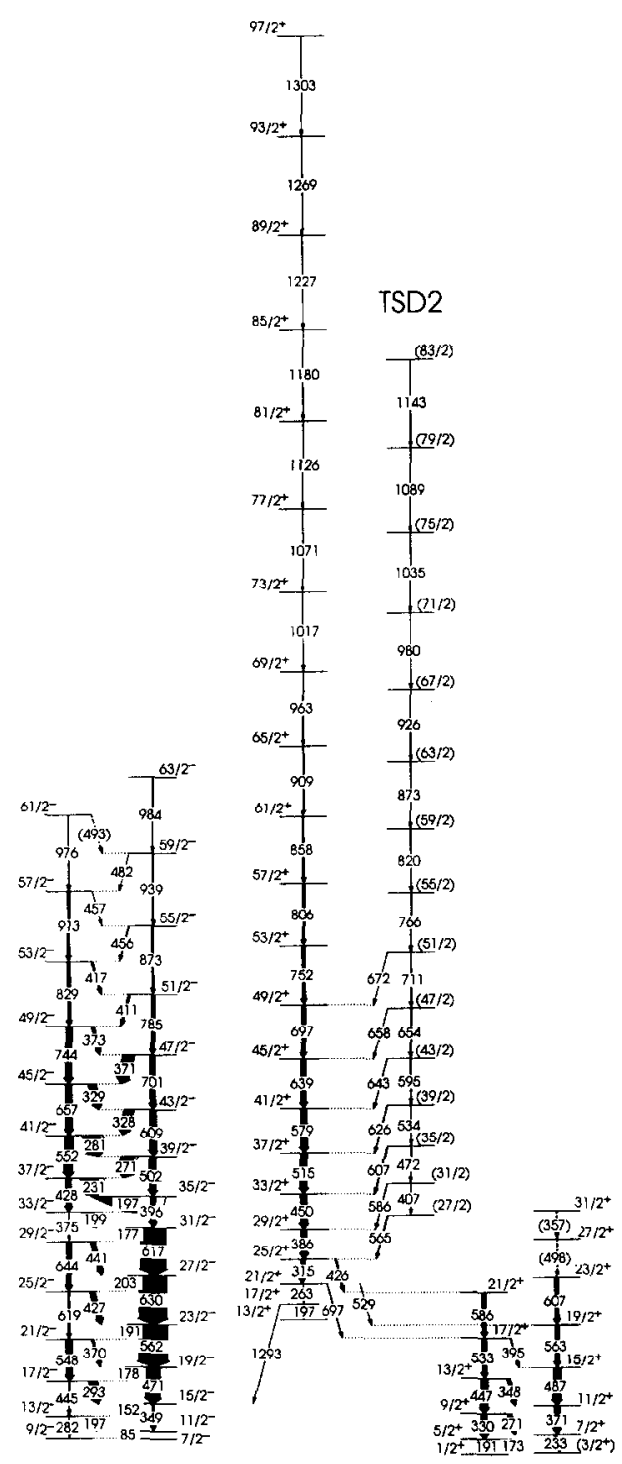

Figure 3. Partial levels scheme of ${ }^{163} \mathrm{Lu}$ showing the two TSD bands together with the linking transitions and the ND structures to which the TSD states decay.

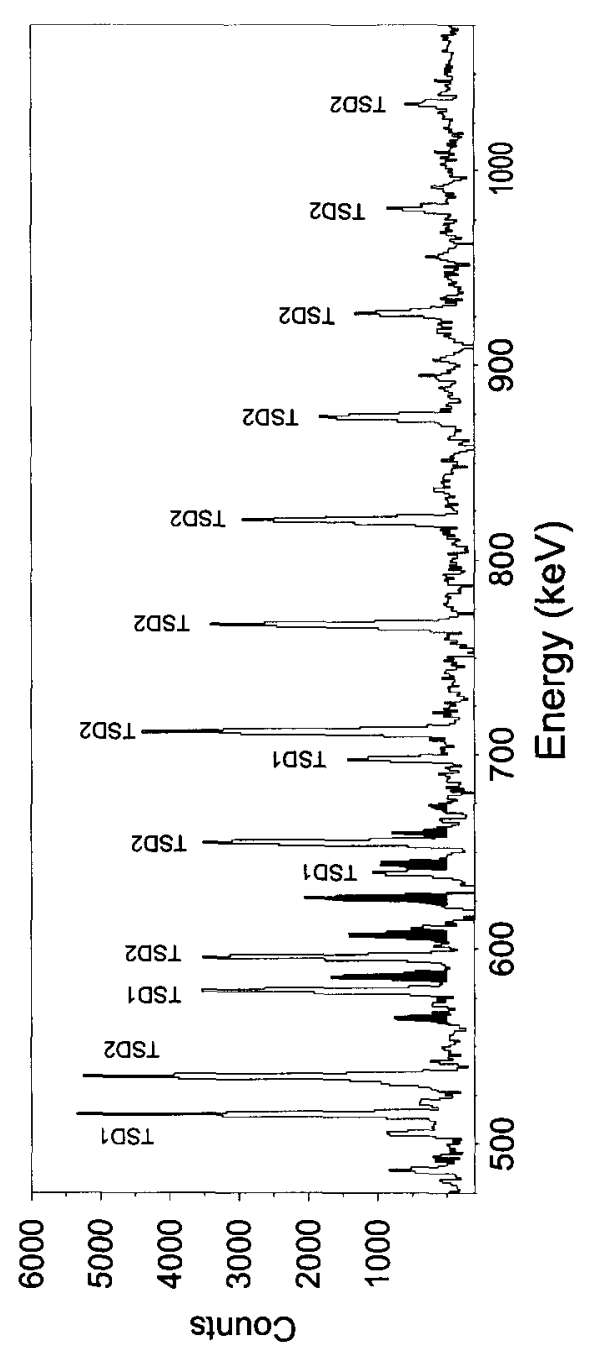

Figure 1. Spectrum of linking transitions, marked in black, between TSD1 and TSD2. The gating conditions are described in the text. 
sible assignments, $(\pi, \alpha)$ for TSD2 are $(+,-1 / 2),(-,-1 / 2)$ and $(+,+1 / 2)$ with $\Delta \mathrm{I}=0 \mathrm{E} 2$ transitions. In the latter case $\Delta \mathrm{I}=2$ branchings to TSD1 of considerably higher strength should have been observed, and this possibility is therefore disregarded.

\section{POSSIBLE CONFIGURATIONS OF TSD2}

A few different possibilities for the configuration of TSD2 exist and must be considered. Calculations using the cranking code the Ultimate Cranker $[8,9]$ predict in addition to the two signatures of the $\pi i_{13 / 2}$ orbital with a large splitting a negative parity orbital, $\pi h_{9 / 2}$ at relatively low excitation and also a large signature splitting. In addition, a wobbling mode built on the aligned $i_{13 / 2}$ proton is suggested, based on particle-rotor model calculations $[10]$.

The most obvious suggestion for the configuration would be the unfavoured signature partner of the $i_{13 / 2}$ proton. However, the experimental signature splitting of $275 \mathrm{keV}$ at $\hbar \omega$ $=250 \mathrm{keV}$, as seen in Fig. 4, is substantially lower than the expected $1400 \mathrm{keV}$ from the cranking calculations. The expected properties for the $\alpha=-1 / 2 \rightarrow \alpha=+1 / 2$ transitions imply almost vanishing $\mathrm{B}(\mathrm{E} 2)_{\text {out }} / \mathrm{B}(\mathrm{E} 2)_{\text {in }}$ and $\mathrm{B}(\mathrm{M} 1) / \mathrm{B}(\mathrm{E} 2)_{\text {in }}$, which is inconsistent with both experimental solutions. In addition, the experimental alignment of TSD2 is larger than the alignment of TSD1, opposite to the expected behavior of signature partners, as they normally split with increasing rotational frequency.

The unfavoured signature partner of the negative parity $h_{9 / 2}$ proton with $\alpha=-1 / 2$ does have the assigned spin values of the levels. However, the fact that TSD2 is $900 \mathrm{keV}$ lower than the expected excitation energy above TSD1, and furthermore, the favoured signature of the $h_{9 / 2}$ configuration is not observed excludes this possibility.

An octupole vibrational excitation would give stretched $\mathrm{E} 1$ multipolarity for the connecting transitions. However, the $\mathrm{B}(\mathrm{E} 1)$ values estimated to $\sim 0.003 e^{2} \mathrm{fm}^{2}$ would be extremely enhanced. At the moment no conclusive information from the angular distribution can be presented and a polarization analysis may be needed to completely exclude this possibility.

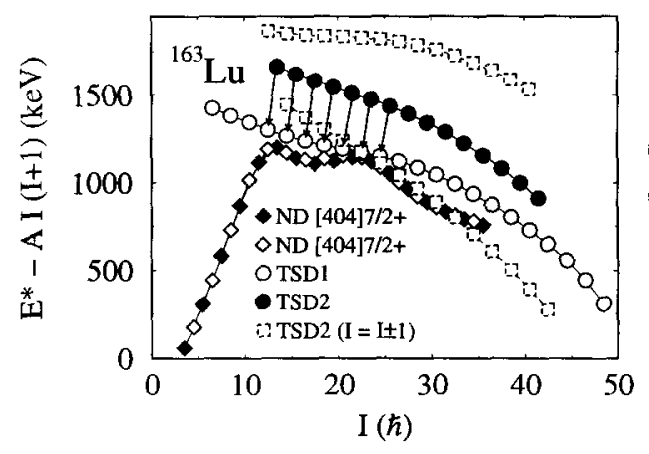

Figure 5. Excitation energies with a rigid rotor reference subtracted.

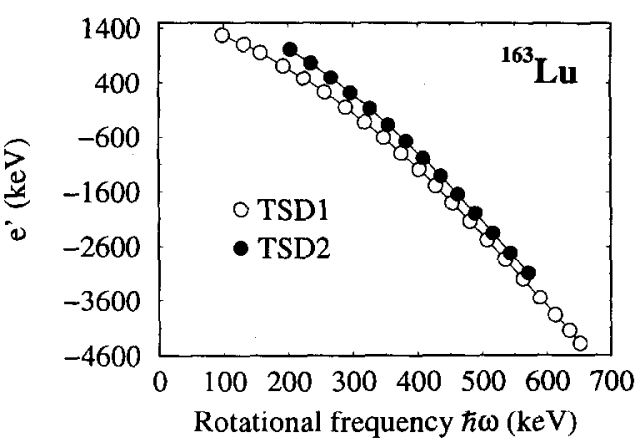

Figure 6. Routhians in ${ }^{163} \mathrm{Lu}$. 
Finally, the most interesting possibility that TSD2 is a wobbling excitation with $n_{w}=1$ built on TSD1 (with $n_{w}=0$ ) is considered. Around the relevant angular momentum region a wobbling mode, $\alpha=-1 / 2$, based on TSD1 can appear energetically lower than the $\alpha=-1 / 2$ signature partner of the $i_{13 / 2}$ proton band, if appropriate values of $\gamma$ and moments of inertia are chosen. Then, the estimated splitting of TSD2 and TSD1 may be a few hundred keV only. Calculated values are $\mathrm{B}(\mathrm{E} 2)_{\text {out }} / \mathrm{B}(\mathrm{E} 2)_{\text {in }} \sim 0.3$ and $\mathrm{B}(\mathrm{M} 1) / \mathrm{B}(\mathrm{E} 2)_{\text {in }} \sim 0.02 \mu_{N}^{2} / e^{2} b^{2}$ for the connecting transitions of mixed E2/M1 nature, in qualitative agreement with the measured DCO ratios and angular distribution analysis. The very similar dynamic moment of inertia for the two TSD bands indicates their similar structure. In the case suggested of TSD2 as a 1-phonon wobbler, the ratio $\hbar \omega_{w} / \hbar \omega_{\text {rot }}$ varies from 1.5 to 0.5 with increasing spin indicating a gradual change in the three moments of inertia.

\section{CONCLUSIONS}

The candidate for a wobbling excitation in ${ }^{163} \mathrm{Lu}$, TSD2, has been connected to TSD1 by 7 linking transitions. The results of the preliminary analysis are in agreement with the assignment of TSD2 as a wobbling excitation, in the presence of an aligned particle, built on TSD1. The alternative interpretation in terms of an octupole vibration is highly unlikely but can at present not be completely excluded.

\section{REFERENCES}

1. W. Schmitz et al., Nucl. Phys. A539 (1992) 112 and Phys. Lett. B 303 (1993) 230.

2. H. Schnack-Petersen et al., Nucl. Phys. A594 (1995) 175.

3. C.X. Yang et al., Eur. Phys. J. A. 1 (1998) 2084.

4. S. Törmänen et al., Phys. Lett. B454 (1999) 8.

5. S. Åberg, Nucl. Phys. A 520 (1990) 35c and references therein.

6. A. Bohr and B.R. Mottelson, Nuclear Structure, Vol. II, (Benjamin, New York, 1975), Chap. 4, p190.

7. J. Domscheit et al., Nucl. Phys. A 660 (1999) 381.

8. T. Bengtsson, Nucl. Phys. A 496 (1989) 56 and A 512 (1990) 124.

9. See R. Bengtsson, http://www.matfys.lth.se/ ragnar/ultimate.html

10. I. Hamamoto, Phys. Lett. B193 (1987) 399. 